\title{
Dislocations in a Ni-based superalloy during low temperature creep
}

\author{
Catherine Rae ${ }^{1}$, Vassili Vorontsov ${ }^{2}$, Libor Kovarik $^{3}$, and Michael Mills ${ }^{4}$ \\ ${ }^{1}$ Department of Materials Science and Metallurgy, Cambridge University, Pembroke Street, Cambridge CB2 3QZ, UK \\ ${ }^{2}$ Department of Materials, Imperial College, South Kensington Campus, London SW7 2AZ, UK \\ ${ }^{3}$ Pacific Northwest National Laboratory, 902 Battelle Boulevard, Richland, WA, USA \\ ${ }^{4}$ Ohio State University, 477 Watts Hall, 2041 College Rd., Columbus, OH 43210, USA
}

\begin{abstract}
The nature and variety of the dislocations passing through the two-phase $\gamma \gamma^{\prime}$ microstructure of Ni-based superalloys is key to the properties of these materials. The chemistry, size and arrangement of the precipitates greatly affects the nature of these dislocations. We present High Angle Annular Dark Field (HAADF) TEM observations of the structure of dislocations entering, passing through the $\gamma^{\prime}$ precipitates in the singlecrystal superalloy CMSX-4®. The creep deformation of the sample was interrupted after 8 hours at $750{ }^{\circ} \mathrm{C}$ and $750 \mathrm{MPa}$, a critical stage just as secondary creep was being established, and shows a range of defects in both phases, not always those predicted by the Schmid factor for the deformation geometry. We show that dislocations lodged in the $\gamma \gamma^{\prime}$ interfaces have a significant effect on the structure of the interface and that they combine to produce stacking faults which cut through the $\gamma^{\prime}$. The implications of these observations for secondary creep deformation are discussed.
\end{abstract}

CMSX-4 $\cap$ is a registered Trade Mark of the Cannon Muskegon Company.

\section{Introduction}

High-resolution techniques are enabling us to look in unprecedented detail at dislocation structures and interactions. Single crystal superalloys are ideal subjects for this as the geometry of deformation can be closely controlled. Their creep strength derives from the regular arrays of ordered $\mathrm{L}_{2}$ precipitates that resist the ingress of regular FCC lattice dislocations [1,2]. At high temperatures the role of the precipitates is largely to exclude dislocations, forcing them to move in the remaining $30 \%$ of $\gamma$ phase by a combination of glide and climb [3]. But in the important low temperature regime, experienced in the hottest part of the engine where the high pressure turbine blades are cooled, dislocations are able to enter the $\gamma^{\prime}$. Furthermore a large part of the strain derives from the cutting of the precipitates by suitable partial dislocations $[4,5]$. The role of stacking fault shear in superalloys has received increasing attention with the focus on high cobalt superalloys that exhibit this mode of behaviour in both tensile and creep deformation [6]. In this paper we examine the microstructure of the alloy CMSX-4® crept to $8 \%$ strain just at the onset of secondary creep. In a previous paper we established the structure of the superlattice partial dislocations, $a / 3\langle 112\rangle$ and also the way in which the lattice dislocations are embedded in the $\gamma \gamma^{\prime}$ interface [7]. In this paper we look at how dislocations combine and contribute to the creep and the implications this has for creep under these conditions.

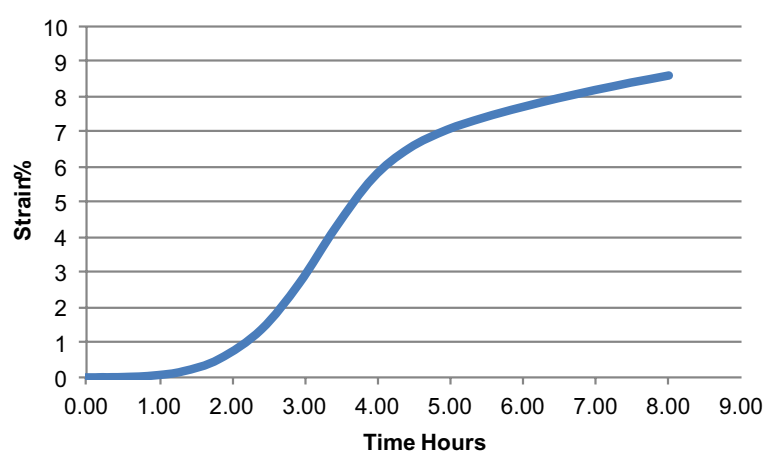

Figure 1. Creep curve: tested at $750^{\circ} \mathrm{C} 750 \mathrm{MPa}$ to $8 \%$ strain as secondary creep is established.

\section{Experimental}

A single crystal test-piece of CMSX-4 was cut with a tensile axis approximately parallel to [001] the deviation from this was $12.7^{\circ}$ from [001] along a plane rotated $24.4^{\circ}$ from (100) around [001]. The sample was crept at $750{ }^{\circ} \mathrm{C}$ $750 \mathrm{MPa}$ and interrupted after $8 \mathrm{~h}$ and at a strain of $8.6 \%$, Fig. 1. The specimen was well into secondary creep when interrupted. The primary slip plane was identified using Laue X-ray imaging and the test-piece sectioned vertically, parallel to the tensile axis. For high resolution imaging the primary slip plane $(-111)$ was perpendicular to the foil normal [110] and the Burgers vector of the highest Schmid Factor [1-12](-111) slip system lies in the plane 

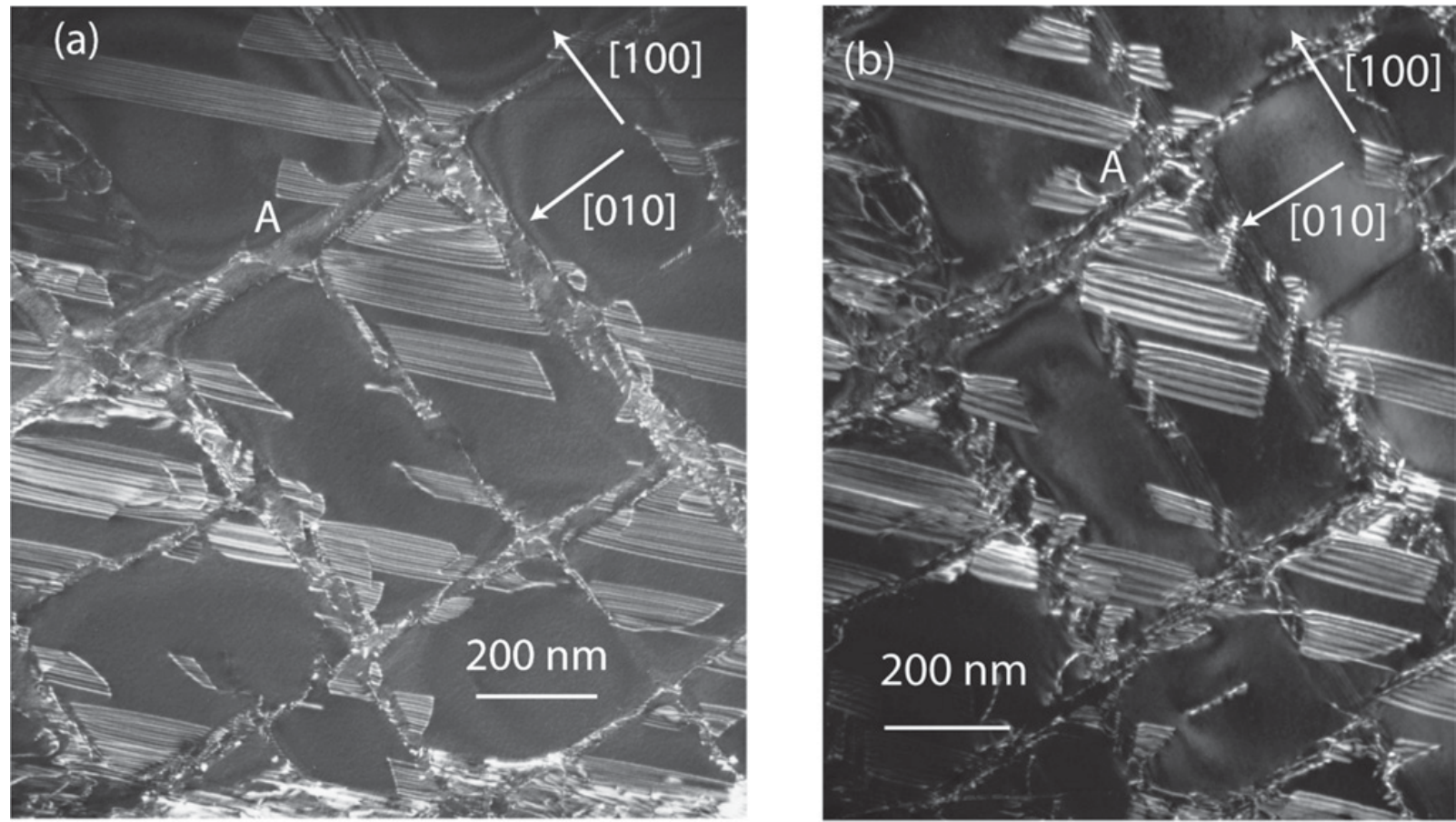

Figure 2. Weak beam micrographs of interrupted microstructure (a) $g=200$, (b) $g=111$. Note the absence of the main dislocation $\mathrm{a} / 2[011]$ in the (100) channel, (a) and $\mathrm{a} / 2[10-1]$ in the (010) channel in (b).

Table 1. Highest four Schmid factors. Starred systems are on primary slip plane for stacking fault shear (-111).

\begin{tabular}{|l|c|c|c|c|}
\hline & 1 & $2^{*}$ & 3 & $4^{*}$ \\
\hline Slip system & {$[011](\overline{1} \overline{1} 1)$} & {$[0 \overline{1} 1](\overline{1} 11)$} & {$[10 \overline{1}](111)$} & {$[101](\overline{1} 11)$} \\
\hline Schmid factor & 0.473 & 0.458 & 0.415 & 0.400 \\
\hline
\end{tabular}

of the foil. Hence an edge dislocation in this system $\mathrm{a} / 3$ [1-12] has its line vector parallel to the foil normal and would be in the ideal configuration to image the atom alignment at high resolution. The sample was also cut on the other vertical section normal to [1-10] and horizontally normal to [001] for imaging the dislocations and stacking faults at lower magnification.

The foils were prepared as described in [7] and imaged in the FEI Titan microscope. Bright and dark field images in two beam conditions were taken using a JEOL 200CX microscope.

\section{Results}

Figure 2 shows a $\{111\}$ TEM two-beam condition of a section cut perpendicular to the [001] tensile axis. Only one active stacking fault system is operative and this is confirmed as being the $(-111)$ plane. This leads to the stacking faults observed in the $\gamma^{\prime}$ and where these terminate in the precipitates some of the Burgers vector are the expected primary slip system a/3[1-12] $(-111)$. Dislocations in the two types of vertical channels each contain principally one type of dislocation: for the (010) channel these have the Burgers vector of $a / 2[10-1]$ and for the (100) channel $b=a / 2[011]$, Fig. 2. Both these dislocations have high Schmid factors but neither on the plane $(-111)$ of the active stacking fault shear: Table 1. However careful examination shows a number of

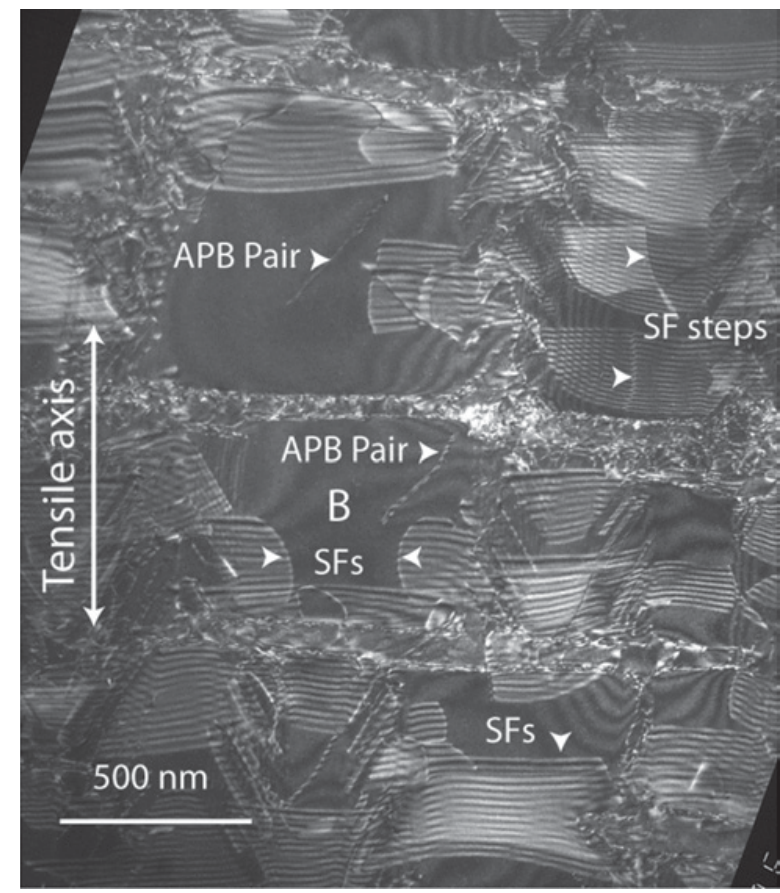

Figure 3. Vertical section viewed along [011]. Note APB paired dislocations and stacking faults emerging from two sides into $\gamma^{\prime}$ to create stepped faults.

examples of other dislocations such as a/2[101] attached to a stacking fault, A, Fig. 2a.

Figure 3 shows a vertical section with a foil normal of $[1-10]$ with the tensile axis vertical. This places the (-111) principal slip plane at $\sim 37^{\circ}$ to the specimen normal. The horizontal $\gamma$ channels, perpendicular to the tensile axis, show the highest density of dislocations forming 
loose networks lying in the $\gamma \gamma^{\prime}$ interface. The vertical channels in this section are at $45^{\circ}$ to the foil and, as in Fig. 2, each contains dislocations of principally one Burgers vector. Multiple stacking faults in each $\gamma^{\prime}$ precipitate lie almost exclusively on the major slip plane in all areas examined, these often exhibit steps. There were also several APB pairs showing a wavy morphology.

\subsection{HAADF of the $\gamma \gamma^{\prime}$ interface}

The structure of $\gamma \gamma^{\prime}$ interface and arrangement of the dislocations in the $\gamma$ channels are key to understanding the dynamics of secondary creep even where the dislocations are penetrating the $\gamma^{\prime}$ precipitates to produce most of the strain [4]. It is clear that the dislocations are principally located in the interface as they are held against this by the imposed shear stress; clear space is visible in the vertical channels, Fig. 2, and the horizontal channels, Fig. 3. Looking at this in high resolution on the (110) vertical section, Fig. 4, gives an edge-on view of the horizontal channel with the principal slip plane normal to the foil. Dislocations cut diagonally across the channel and are embedded in the interface dissociated into two Shockley partials. The line vector of these dislocations is [110], the intersection of the slip plane $(-111)$ and the $\gamma \gamma^{\prime}$ interface (001). Frank circuits around the dislocations show failure closure consistent with Burgers vectors $\mathrm{a} / 2[101]$ or $\mathrm{a} / 2[0-11]$, both of which have the same edge component, a/4 [1-12]. The screw components are equal and opposite, normal to the plane of view and not visible. The $\gamma \gamma^{\prime}$ interface is distorted around the dislocations, such that the misfit between the phases acts to compensate the strain field associated with the edge component of the Burgers vector. The $\gamma$ phase is the brighter side as it contains more atoms of high atomic number. It has the larger lattice parameter in this negative misfit alloy and lies on the tensile side of the dislocation. Other dislocations common in the channels cut the horizontal channel perpendicular to the viewing directions. These will not be visible in this section except as a blurring of the image as they misalign the atom columns.

The dislocations enter the $\gamma$ channels as looped dipoles constrained by the precipitates. Hence one would expect them to lie on the same slip plane. Extrapolating the slip plane (vertical) in Fig. 4 shows they are not. Hence despite the separation of the partials by about $14 \mathrm{~nm}$ and the adjustment of the interface position and chemistry in response to the strain field of the dislocation, climb of the dislocations in the $\gamma \gamma^{\prime}$ interface has occurred. Of the four dislocations with the highest Schmid factors, two lie on the $(-111)$ slip plane and their Burgers vectors add to make up the leading dislocations of the stacking fault.

$$
\begin{aligned}
\mathrm{a} / 2[101]+\mathrm{a} / 2[0-11] & \rightarrow \mathrm{a} / 2[1-12] \\
& \rightarrow \mathrm{a} / 3[1-12]+\mathrm{a} / 6[1-12] .
\end{aligned}
$$

These dislocations combine by climb in the interface: the first reaction involves an increase in the total line energy, a reduction coming only after the second split. Figure 5 gives some insight into this process. Frank circuits around each dislocation show both dissociated on the

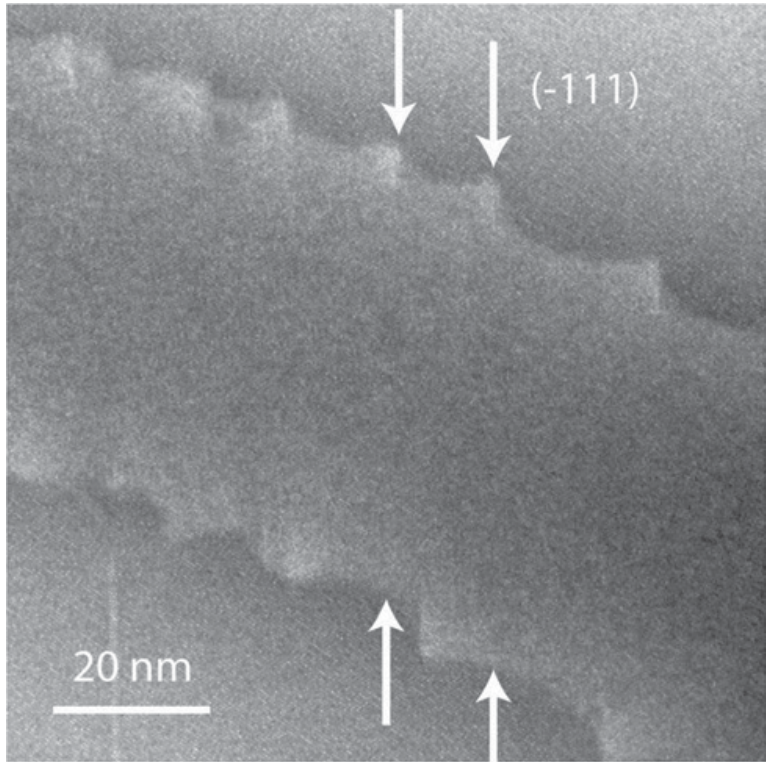

Figure 4. HAADF image of horizontal channel showing the Cusps at dissociated dislocations in the interface. Note the misalignment of the dislocations on each side.

(-111) with plane edge components a/4 [1-12], a circuit around both giving $\mathbf{b}_{\text {edge }}=\mathrm{a} / 2$ [1-12]. Hence we infer that they are $\mathbf{b}=\mathrm{a} / 2$ [101] and $\mathrm{a} / 2$ [0-11]. At first it was thought the fault on the LHS lay on the line of strong contrast; closer examination revealed that there is no structural fault at this position and it had moved some 16 close packed planes to the right, marked by the two arrows. This suggests that the final part of the combination occurs by the dislocation on the left climbing without taking the elemental segregation with it. The stacking fault width is about $6 \mathrm{~nm}$, that on the right $15 \mathrm{~nm}$, that of the other dislocations being typically $4 \mathrm{~nm}$.

From these images it is not possible to confirm that the screw components are opposite, but it would be hard to understand why the dislocations were approaching each other if they had the same Burgers vector. As the two dislocations are dissociated on the same plane, this is not a section through a node in a network on the $\gamma \gamma^{\prime}$ interface two dislocations crossing on the horizontal interface would necessarily be on different planes.

After combining to produce the dislocation a/2 [1-12] at the interface, a dislocation of $\mathrm{a} / 3$ [1-12] can move into the $\gamma^{\prime}$ trailed by a SISF, as seen in Fig. 6. The cusp in the $\gamma \gamma^{\prime}$ interface remains close to the position of the Shockley partial a/6 [1-12] marked by the arrow.

\subsection{HAADF of Stacking faults in $\boldsymbol{\gamma}^{\prime}$}

In this section we focus on the dislocation combinations in the $\gamma^{\prime}$. Both intrinsic and extrinsic stacking faults are seen and they can be readily distinguished in the HAADF image by the number of planes displaced in the fault and also by the pattern of segregation to the fault, Fig. 7. There are more intrinsic faults seen than extrinsic consistent with previous work on orientations near $\langle 001\rangle$, [9].

Many examples of closely-spaced SISFs were seen; some very close, others further apart, Fig. 3. In Fig. 8 

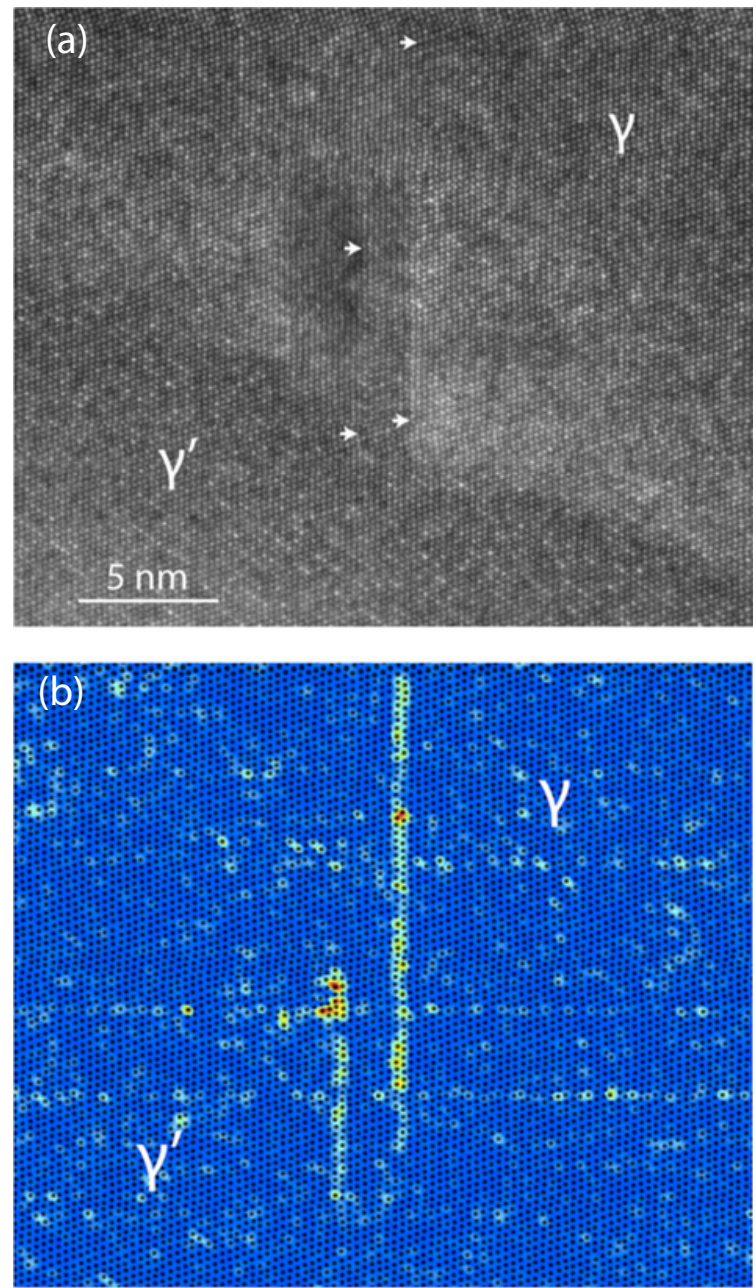

Figure 5. (a) Image of two dislocations in the $\gamma \gamma^{\prime}$ interface merging. The position of the Shockley partials at each end off the dissociated dislocations are marked with arrows. (b) Centre of symmetry image to show fault positions (in preparation, placeholder in position).

two SISFs have come to a halt on parallel planes. Frank circuits around the end of each fault gives a Burgers vector of $\mathrm{a} / 6[1-12]$. An edge Shockley partial inside the ordered $\gamma^{\prime}$ would require an APB fault beyond the SISF. Following the brighter streaks on alternate (001) planes in Fig. 8b indicates an APB joining the two terminating dislocations. The exact position is difficult to determine as the streaking becomes very indistinct in this area. This could have been formed by the climb of an a/2[101] or $\mathrm{a} / 2$ [01-1] dislocations between the partial. If no APB fault is seen it is more likely that the terminating faults are either $\mathrm{a} / 3$ [211], or $\mathrm{a} / 3[-1-21]$, both of which lie in the $(-111)$ slip plane and have a projected Burgers vector of $a / 6$ [1-12] in the viewing direction as observed. These Burgers vectors have a screw component of $\pm \mathrm{a} / 2[110]$ respectively and could glide between the partials to produce an APB, but this would not give a visible fault as the displacement would be in the viewing direction. An exchange of dislocations by whichever mechanism would effectively lock the dislocations giving a visible step.

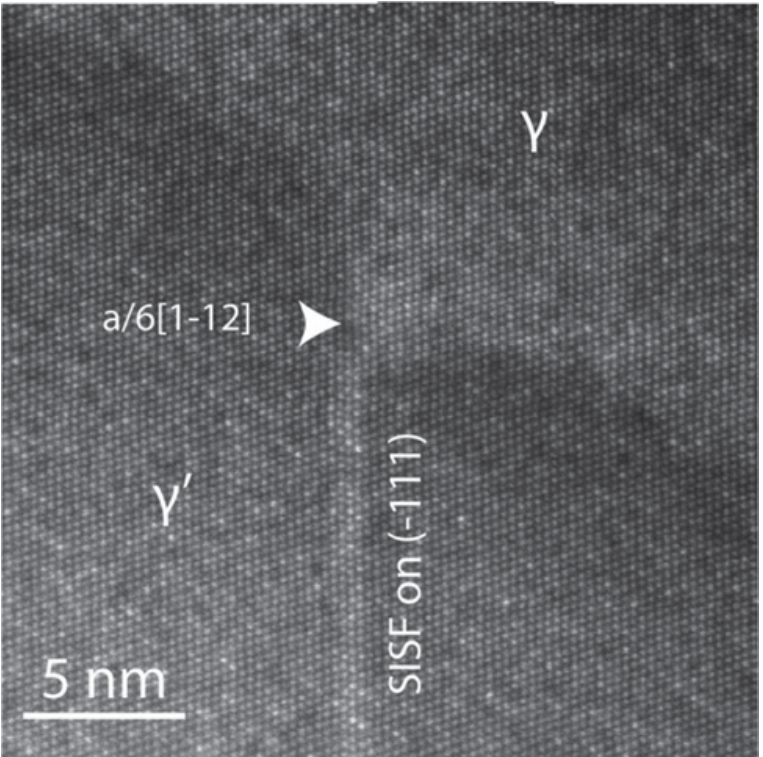

Figure 6. HAADF image of a SISF, terminating at the $\gamma \gamma^{\prime}$ interface in a Shockley partial, following the combination of two lattice dislocations in the interface.

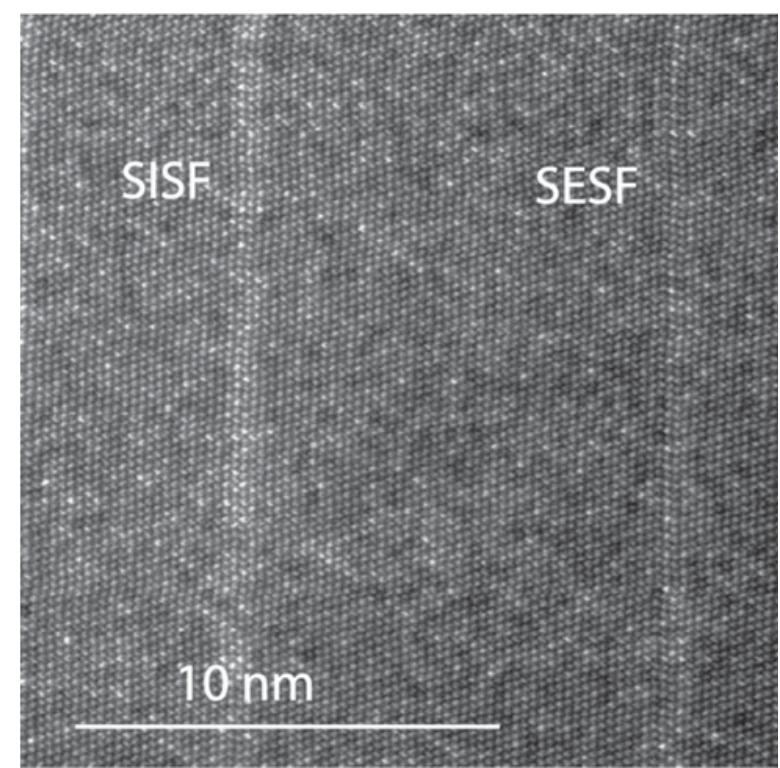

Figure 7. HAADF image of a SISF (left) and a SESF fault (right) showing the change in contrast at the faults.

This configuration could have been formed by a single fault bisected by several other dislocations causing a displacement by several lattice planes; alternatively, two similar partials could have arrived from opposite sides of the precipitate on closely parallel planes, the interaction of the strain fields capturing the two partials as a dipole. Splitting the faults by cutting is unlikely, as there is very little activity on other slip planes in the $\gamma^{\prime}$, and the displacement is not consistent across adjacent faults. The second possibility, bypass and capture, could result from the entry into the $\gamma^{\prime}$ of two similar SISFs led by $\mathrm{a} / 3$ [1-12] and trailed by either $\mathrm{a} / 3$ [211] or $\mathrm{a} / 3[-1-21]$. If they were on closely parallel planes the leading partials would experience a strong force from the applied force, 

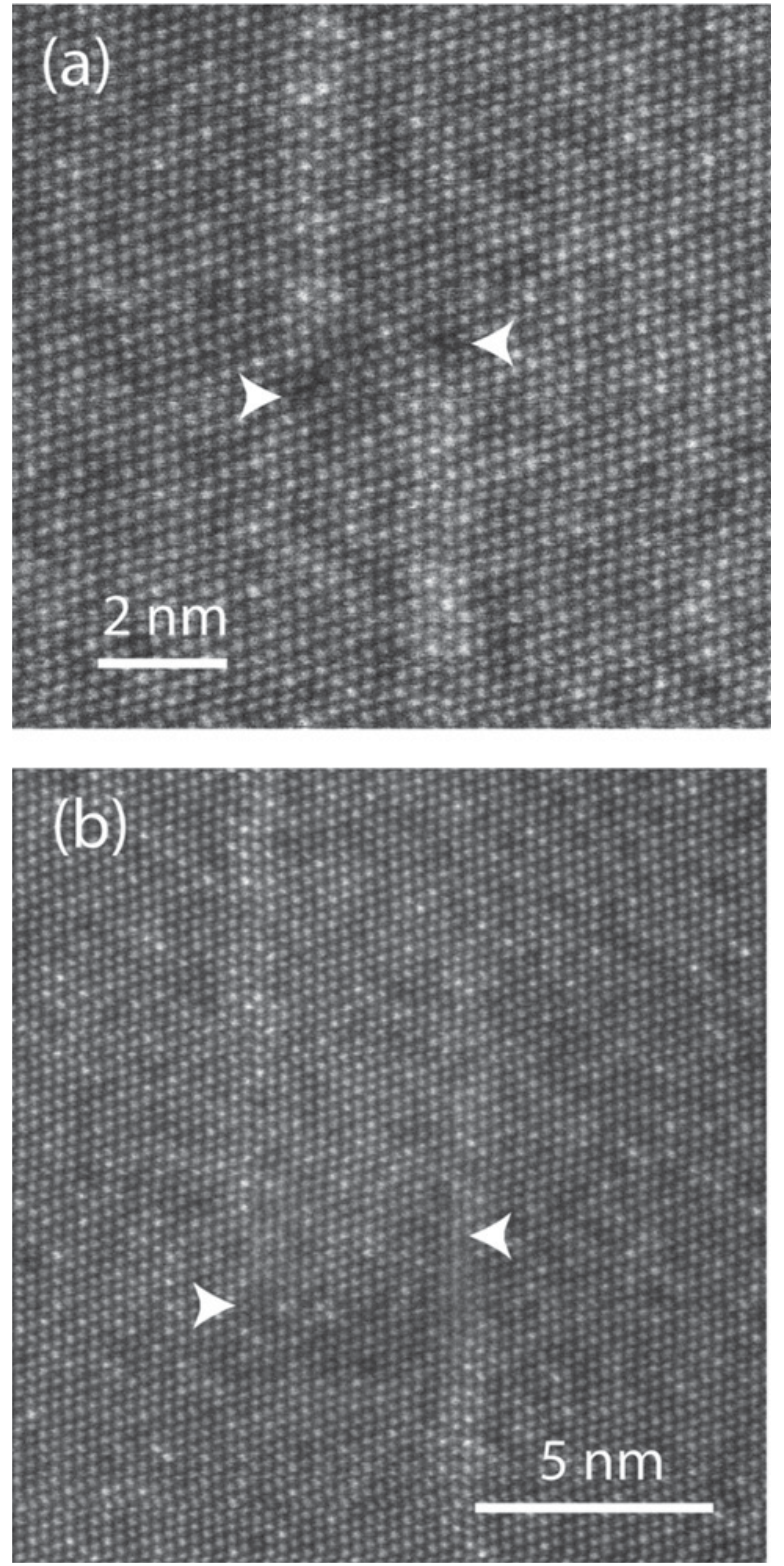

Figure 8. Closely spaced SISF stacking faults in the $\gamma^{\prime}$ : (a) 9 layers apart; (b) 18 layers apart with an APB fault located between the partials (marked with arrows). Note the fuzzy contrast below the left dislocation indicating it is not aligned with the [110] viewing direction.

twice that on the trailing partials. These would have equal and opposite screw components which could cross-slip and annihilate leaving an APB between the faults. This is consistent with the configurations in Fig. 8.

A potential development of this is the "zig-zag" SISF configuration shown in Fig. 9. A Frank circuit around both the faults shows no closure failure in the plane of the image, consistent with a dipole. Following from the configuration in Fig. 9, the passage of a further Shockley partial a/6[-112] would produce an SISF on the plane joining the SISFs and $\pm \mathrm{a} / 3[002]$ stair-rod dislocations at the corners. These have distinctive patterns of segregation to the two corners. This is summarised in the diagram in Fig. 10.

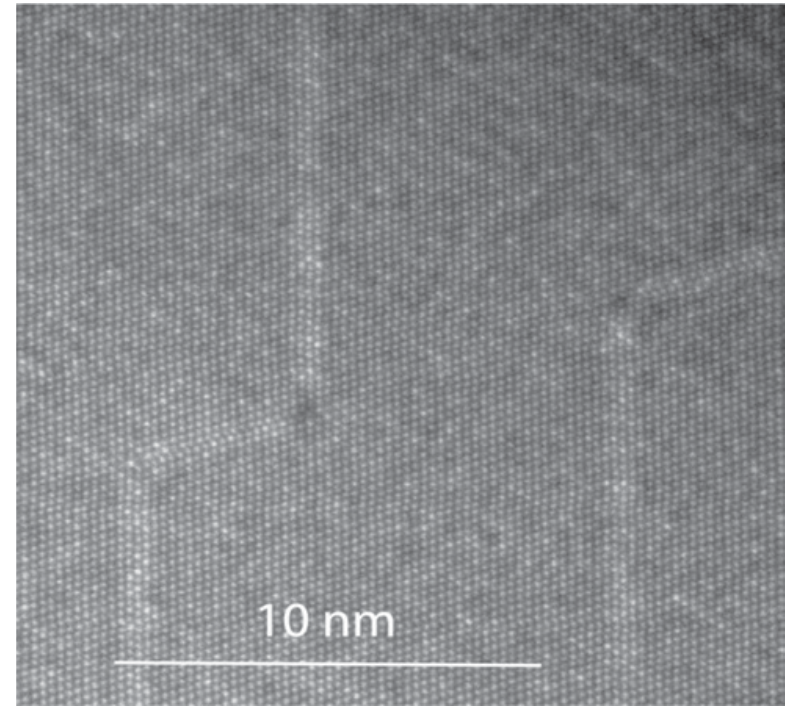

Figure 9. HAADF image of several SISF faults linked by fully developed stacking faults showing bright contrast. Note the joining faults are not aligned along the slip plane.

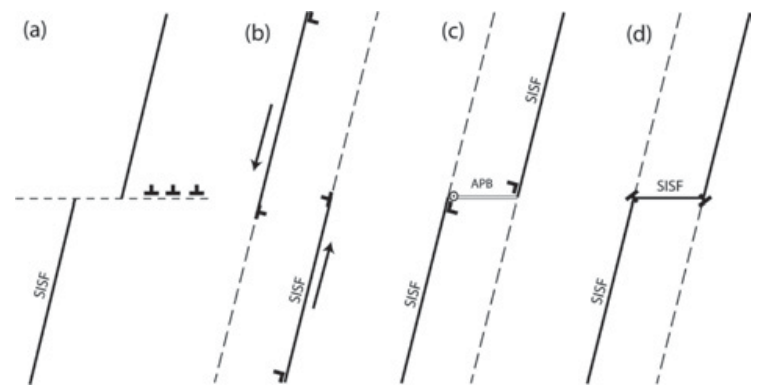

Figure 10. (a) SISF cut by dislocations to give step. (b) Two parallel SFs pass, (c) trailing partials locked and screw dislocation passes on conjugate plane, (d) partial transmitted to give zig-zag defect of SISF.

\section{Discussion}

The observations described give some insight into the mechanisms of secondary creep. After $8 \%$ deformation dislocations have reached almost all parts of the gamma as constrained loops producing the highest concentrations in the horizontal channels where at least four different Burgers vectors form complex networks. In the vertical channels dislocations are less dense and are predominantly, but not exclusively, of a single Burgers vector. In the case here the two most common Burgers vectors are $a / 2[10-1]$ and $a / 2[011]$. Neither of these contributes to the superlattice partials necessary to produce the stacking faults observed on the (-111) plane. However the two systems, a/2[0-11](-111) and a/2[101](-111), which combine to produce the Burgers vector $\mathrm{a} / 2[1-12]$, have the $2^{\text {nd }}$ and $4^{\text {th }}$ highest Schmid factor, are present and are coplanar. There are several examples of these opening up into stacking faults and entering the $\gamma^{\prime}$ in Fig. 2 at A.

The macroscopic effect of climbing networks of dislocations in the $\gamma \gamma^{\prime}$ interface, emitting SISFs into the $\gamma^{\prime}$ when the appropriate dislocations meet, provides a mechanism for secondary creep consistent with the strain evolution, [4]. Strain comes from both the climb of 
dislocations around the $\gamma^{\prime}$ precipitates, as at higher creep temperatures [2], and the shear of the $\gamma^{\prime}$ by superlattice partials. The continued distortion of the lattice consistent with stacking fault shear reported in [4] suggests that the shear of the gamma prime, facilitated by climb in the interface, is the major component of the strain. The activation energy for primary creep would be determined by the diffusion-controlled re-ordering of the superlattice partial dislocations forming the stacking faults. The critical step in secondary creep becomes the supply of faults controlled by climb in the $\gamma \gamma^{\prime}$ interface rather than their movement giving an activation energy similar to creep at higher temperatures where shear is absent $[4,10]$.

The important first step in the creep appears to be the combination of a single pair of dislocations at $60^{\circ}$ to give an a/3[1-12] partial which can move in the $\gamma^{\prime}$ to leave a SISF and a Shockley partial trapped at the interface. This trailing dislocation can be released to cause further shear by a number of interactions with other dislocations climbing in the $\gamma \gamma^{\prime}$ interface. One such described above is the combination with an a/2[110] to give a trailing dislocation at $60^{\circ}$ from the leading super-partial. Although this dislocation has a very low Schmid factor (0.057) for this orientation, it can be formed by combination of the two most common dislocations, typically as part of a network, $a / 2[10-1]+a / 2[011]$. Overall, this is the dissociation of either $\mathrm{a}[10-1]$ or $\mathrm{a}[011]$ perfect dislocation into two superpartials. However the evidence suggests that this dissociation does not happen spontaneously. In an orientation containing only two sets of dislocations with Burgers vectors at $90^{\circ}$, no stacking faults were formed and the creep life was exceptional [10]. Such a reaction forcing two equal dislocations together would require a greater activation energy than the initial combination of pair at $60^{\circ}$.

Many other mechanisms are possible, for example two further dislocations the same as the initial pair, give the stacking fault ribbon seen in the early stages of creep [12]. This would produce SESFs as occasionally seen. It is clear that there is a release mechanism for the Shockley partials, otherwise the stacking fault density would build up throughout creep, contrary to observation [13].

The observation of several closely spaced pairs of stacking faults or stepped stacking faults suggests that some of these events are related. Take for example the two stacking faults emerging from the vertical channels on very similar planes at B in Fig. 3. Two stacking faults are emerging (or receding) from opposite sides of a single precipitate essentially on the same plane and with the same Burgers vector. At this scale it is impossible to judge whether, when they meet, they will be in perfect alignment or closely parallel. The coincidence of two dislocations emerging independently from the sides of the same precipitate on almost the same plane is unlikely. However if one of the dislocations necessary for the main stacking fault partial is relatively common - the a/2[0-11] with the second largest Schmid factor - and the other a/2 [101] is much rarer in the gamma channels, the trigger for a SISF able to enter the $\gamma^{\prime}$ is likely to be the arrival of the latter dislocation wrapping around the precipitate. Interaction requires climb, and the horizontal channels, already highly populated by closely spaced networks of dislocations, offer an effective barrier to penetration. The vertical channels however have a lower density and the absence of networks allows the newly formed stacking fault to enter the $\gamma^{\prime}$. Hence it enters on either side at the vertical channels. In climbing to find suitable pairing dislocations, the plane of entry is slightly changed. Hence when the two faults enter the $\gamma^{\prime}$ and move together to complete the stacking fault they will be on parallel planes leading to the variety of closely paired stacking faults and stepped stacking faults seen at high resolution, Figs. 8 and 9. These are also quite common at lower resolution and often curved. The curvature would prevent the formation of a stacking fault on the linking plane as in Fig. 9. Examples are labelled in Fig. 3.

\section{Conclusions}

The alloy CMSX-4 was crept at $750{ }^{\circ} \mathrm{C}$ and $750 \mathrm{MPa}$ to $8 \%$ strain, in the early stages of secondary creep. Observations were made using conventional two beam and high resolution HAADF TEM to investigate the dislocation reactions.

1. Arrays and networks of dislocations populate all the $\gamma \gamma^{\prime}$ interfaces, these distort the interface and show evidence of segregation.

2. Dislocations are observed to climb in the interface and combine to produce SISF faults in the $\gamma^{\prime}$.

3. The remaining Shockley partials combine with other dislocations to continue the shear removing the stacking fault.

4. SISFs often penetrate from opposite sides of a precipitate becoming entrapped to produce steeped faults or zig-zag faults.

5. Strain is produced by stacking fault shear, but the creep rate is controlled by dislocations in the $\gamma \gamma^{\prime}$ interface.

CR and VV would like to acknowledge Rolls-Royce plc and the EPSRC for financial support for this project under EP/H022309/1 and EP/H500375/1. LK and MJM acknowledge the support of GE Aviation under the University Strategic Alliance program. We also acknowledge the use of TEM facilities at Cambridge University, and CEMAS at the Ohio State University.

\section{References}

[1] T.M. Pollock and A.S. Argon, Acta Metal. Mater. 40, 1-30 (1992)

[2] N. Matan, D.C. Cox, P. Carter, M.A. Rist, C.M.F. Rae, R.C. Reed, Acta Mater. 47, 1549-1563 (1999)

[3] N. Matan, D.C. Cox, C.M.F. Rae and R.C. Reed, Acta Materialia, 47, 2031-2045 (1999)

[4] G.L. Drew, R.C. Reed, K. Kakehi, and C.M.F. Rae, Superalloys, Eds K.A. Green et al, The Minerals Metals and Materials Society, 127-136 (2004).

[5] C.M.F. Rae and R.C. Reed, Acta Materialia, 55 1067-1081 (2007)

[6] A. Suzuki, G.C. DeNolf and T. M. Pollock, Scripta Mat. 56, 385-388 (2007)

[7] V.A. Vorontsov, L. Kovarik, M.J. Mills, C.M.F. Rae, Acta Materialia, 60, 4866-4878 (2012)

[8] P. Caron, T. Khan, Mater. Sci. Eng. 61173 (1990) 
[9] Q.Z. Chen and D.M. Knowles, Materials Science and Engineering, A356, 352-367 (2003)

[10] G.R. Leverant and B.H. Kear, Metall. Trans.,1, 491 (1970)

[11] C.M.F. Rae, N. Matan, DC Cox, MA Rist, R.C. Reed. Met. Trans. A, 31, 2219-27 (2000)
[12] C.M.F. Rae, N. Matan, R.C. Reed. Mater Sci Eng A; 300:125-34, (2001)

[13] V. Sass, U. Glatzel and M. Feller-Kniepmeier, Superalloys 1996, Eds R.D. Kissinger et al, The Minerals Metals and Materials Society, 283-290 (1996) 